Hi gh- speed at omi c for ce mi croscopy for observing protei $n$ mol ecul es in dynami $c$ action

\begin{tabular}{|l|l|}
\hline 著者 & Ando Toshi o \\
\hline $\begin{array}{l}\text { j our nal or } \\
\text { publ i cat } \mathrm{i} \text { on } \mathrm{t} \text { i t l e }\end{array}$ & $\begin{array}{l}\text { Proceedi ngs of SPI E - The I nt er nat i onal } \\
\text { Soci et y f or Opt i cal Engi neer i ng }\end{array}$ \\
\hline vol une & 10328 \\
\hline page $r$ ange & $103281 R$ \\
\hline year & $2017-01-01$ \\
\hline URL & ht t p: //hdl . handl e. net /2297/47884 \\
\hline
\end{tabular}




\title{
High-speed Atomic Force Microscopy for Observing Protein Molecules in Dynamic Action
}

\author{
T. Ando ${ }^{1,2}$ \\ ${ }^{1}$ Bio-AFM Frontier Research Center, Institute of Science and Engineering, Kanazawa University, Kakuma-machi, \\ Kanazawa 920-1192, JAPAN \\ ${ }^{2}$ CREST, Japan Science and Technology Agency, Sanban-cho, Chiyoda-ku, Tokyo 102-0075, JAPAN
}

\begin{abstract}
Directly observing protein molecules in dynamic action at high spatiotemporal resolution has long been a holy grail for biological science. To materialize this long quested dream, I have been developing high-speed atomic force microscopy (HS-AFM) since 1993. Tremendous strides were recently accomplished in its high-speed and low-invasive performances. Consequently, various dynamic molecular actions, including bipedal walking of myosin $\mathrm{V}$ and rotary propagation of structural changes in $\mathrm{F}_{1}$-ATPase, were successfully captured on video. The visualized dynamic images not only provided irrefutable evidence for speculated actions of the protein molecules but also brought new discoveries inaccessible with other approaches, thus giving great mechanistic insights into how the molecules function. HS-AFM is now transforming "static" structural biology into dynamic structural bioscience.
\end{abstract}

Keywords: High-speed AFM, proteins, dynamic processes, high frame rate, atomic force microscopy

\section{INTRODUCTION}

Proteins are structural and functional elements of life. Nearly all biological phenomena, such as muscle contraction, cell division and even thinking and memory, are all proteins' works. Understanding how proteins work to produce complex biological architectures and phenomena is one of central issues in life-sciences. The most widely used experimental approach to this end has been atomic structure determination of proteins by X-ray crystallography. The most recent advancement of cryo-electron microscopy has also allowed atomic structure determination for proteins embedded in ice, thanks to the sensitive camera capable of direct electron detection. NMR has also been successfully used to obtain the atomic structure of proteins in solution and even in living cells. Despite these advancements, the acquirable structural information is limited to static snapshots of proteins. Single-molecule biophysical techniques have been devised to observe dynamic behavior of optical markers attached to protein molecules. And yet, the protein molecules themselves are invisible even with super-resolution.

In 1986, atomic force microscopy (AFM) was invented to visualize atoms on solid surfaces ${ }^{(1)}$. AFM is also applicable to objects on a surface in solution. This unique capability has been widely exploited to directly observe protein molecules in physiological solution. In 1992, Gerd Binnig, a co-inventor of AFM, predicted as "In biology, use of the force microscope will probably become quite common because of its ability to deliver films of processes"(2). However, it takes a minute or more to acquire an AFM image, too slow to film biological processes in real time. To materialize high-speed AFM (HS-AFM), I embarked on the development of underlying techniques around 1993. I built the first prototypic HS-AFM setup in $2001^{(3)}$ and finally established HS-AFM of practical use in $2008^{(4)}$. The following briefly describe a theoretical consideration of imaging rate of AFM, elementary techniques contained in HS-AFM, and HSAFM imaging of a motor protein, myosin V.

\section{IMAGING RATE OF AFM}

What factors do determine the highest possible imaging rate of AFM? Since the imaging rate depends on the imaging mode, we here consider only the amplitude modulation mode that is often used for biological samples. In this mode, the cantilever is oscillated at (or near) its first resonant frequency $f_{\mathrm{c}}$ so that the oscillating cantilever tip intermittently taps the sample surface. Because of the intermittent tip-sample contact, dragging forces are hardly exerted from the tip to the sample during lateral sample stage scan. During imaging, the oscillation amplitude is managed to be maintained constant by feedback control. For simplicity, let's assume that the sample has a sinusoidal shape in the XZ plane, characterized with a periodicity $\lambda$ and a peak height $h_{0}$. When this sample is scanned in the X-direction with velocity $V_{\mathrm{s}}$ and the feedback controller is switched off, the sample height $h$ under the cantilever tip changes with time as

$$
h(t)=\frac{1}{2} h_{0} \sin (2 \pi f t)
$$


where $f \equiv V_{\mathrm{s}} / \lambda$ (black line, Fig. 1a). When the feedback controller is switched on, the Z-scanner is moved sinusoidally at the feedback frequency $f$ in the direction opposite to the sample height (red line, Fig. 1a). However, because of the chasing-after nature of feedback control, the Z-scanner moves with a time delay $\tau_{0}$ as

$$
Z(t)=-\frac{1}{2} h_{0} \sin \left(2 \pi f t-\theta_{0}\right)
$$

where $\theta_{0} \equiv 2 \pi f \tau_{0}$ is the phase delay of feedback control. Because of this delay, we always have a feedback error $\Delta Z(t)=h(t)+Z(t)$ (blue line, Fig. 1a), which is given by

$$
\Delta Z(t)=h_{0} \sin \left(\theta_{0} / 2\right) \cos \left(2 \pi f t-\theta_{0} / 2\right)
$$

The feedback bandwidth $f_{\mathrm{B}}$ is usually defined by the feedback frequency at which $\pi / 4$ phase delay occurs, and hence, given by $f_{\mathrm{B}}=1 /\left(8 \tau_{0}\right)$. Supposing that the

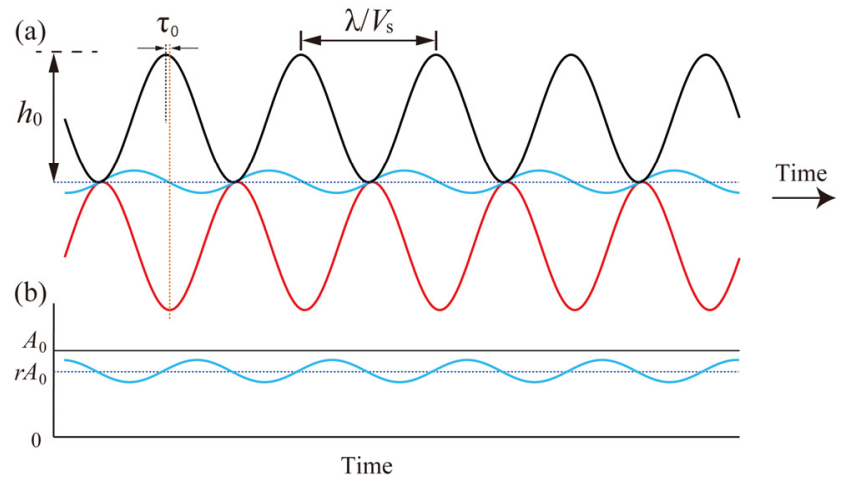

Fig. 1. (a) Z-scanner movement tracing sinusoidally shaped sample that is being scanned in the X-direction with velocity $V_{\mathrm{s}}$. Black line, sample height change under the tip; red line, Zscanner trajectory; blue line, tracing error. (b) Change of cantilever oscillation amplitude (blue line).

phase delay up to $\pi / 4$ has no practical problem for the sample, the X-scanner can be scanned at the highest rate $V_{\mathrm{s}}^{\max }=$ $\lambda f_{\mathrm{B}}$. Therefore, in this case the highest possible imaging rate $R_{\max }$ is given by

$$
R_{\max }=V_{s}^{\max } /(2 W N)=\lambda f_{B} /(2 W N)
$$

where $W$ is the scan range in the $\mathrm{X}$-direction and $N$ is the number of scan lines.

The feedback controller attempts to maintain the cantilever oscillation amplitude $A$ constant at its set point $A_{\mathrm{S}} \equiv r A_{0}$ ( $A_{0}$, cantilever's free oscillation amplitude; $\left.0<r<1\right)$. But, in reality, $A$ approximately changes as $A=r A_{0}-\Delta Z(t)$ (see the blue line in Fig. 1b). Therefore, a tip-force larger than the set point force corresponding to $r A_{0}$ is exerted to the sample when its uphill region is being scanned, whereas a tip-force smaller than the set point force is exerted when the downhill region is being scanned. When the excessive force is too strong to the sample, the phase delay has to be reduced by slowing the scan speed $V_{\mathrm{s}}$ down. Therefore, the largest possible phase delay $\theta_{\max }$ depends on the sample fragility. Considering this issue, Eq. 4 is rewritten as

$$
R_{\max }=2 \theta_{\max } \lambda f_{B} /(\pi W N)
$$

For example, under a realistic condition for imaging protein molecules by our HS-AFM $\left(f_{\mathrm{B}}=110 \mathrm{kHz}, \theta_{\max }=\pi / 9\right.$ (i.e., $20^{\circ}$ ), $\lambda=10 \mathrm{~nm}, W=150 \mathrm{~nm}$ and $N=100$ ), Eq. 5 gives $R_{\max }=16.3$ frames per second (fps). The condition $\theta_{\max } \approx \pi / 9$ generally holds for proteins, according to our experiences. Note that $R_{\max }$ depends on the spatial frequency of the sample surface corrugation to be detected, $1 / \lambda$. To reduce the excessive tip force, we can increase the set point amplitude $A_{\mathrm{s}}$ (i.e., $r$ is set closer to 1 ), instead of slowing the scan speed dwon. However, this setting has a limitation. The amplitude $A=r A_{0}-\Delta Z(t)$ cannot overstep the range of $0<A<A_{0}$ (see Fig. 1b). The curve shown with the blue line in Fig. $1 \mathrm{~b}$ is lifted up by setting $r$ closer to 1 but should always be below the line $A=A_{0}$. This condition gives the following relationship:

$$
r A_{0}+h_{0} \sin \left(\theta_{0} / 2\right)<A_{0}
$$

When this condition is broken, the tip-sample contact is lost, upon which $A$ becomes $A_{0}$ and hence the feedback error signal gets saturated at a small value, $A_{0}(1-r)$. Because of this small saturated error, it takes a long time for the detached tip to land on the sample surface again, like parachuting. For example, for $A_{0}=h_{0} / 5$ and $r=0.9$, Eq. 6 limits the phase delay as $\theta_{0}<2.3^{\circ}$, which severely limits the feedback frequency $f$ as $f<0.05 f_{\mathrm{B}}$. Therefore, without solving this parachuting problem, HS-AFM that can perform low-invasive imaging of fragile biological samples could not be achieved.

\section{TECHNIQUES DEVELOPED FOR HS-AFM}

To increase the imaging rate of AFM, we first have to minimize the time delay of feedback control by increasing the response speed of all devices (the mechanical devices in particular) contained in the feedback loop. Second, we have to minimize unwanted vibrations that are generated when the scanner is displaced at high frequencies. Third, we have to make high-speed imaging compatible with low-invasive imaging. The following are techniques we developed to meet these three demands (Fig. 2). 


\subsection{Small Cantilevers}

The cantilever's response time in tapping mode is given by $Q_{\mathrm{c}} /\left(\pi f_{\mathrm{c}}\right)$, where $Q_{\mathrm{c}}$ and $f_{\mathrm{c}}$ are cantilever's quality factor in water and resonant frequency in water, respectively. To increase $f_{\mathrm{c}}$, while maintaining the spring constant $k_{\mathrm{c}}$ small, the cantilever has to be miniaturized ${ }^{(5)}$. We developed small $\mathrm{Si}_{3} \mathrm{~N}_{4}$ cantilevers of $7 \mu \mathrm{m}$ long, $2 \mu \mathrm{m}$ wide and $90 \mathrm{~nm}$ thick (shown within the white circle in Fig. 2b), by collaborating with Olympus ${ }^{(3,6)}$. Their mechanical properties are $f_{\mathrm{c}}=1.2$ MHz in water, $Q_{\mathrm{c}}=2$ in water and $k_{\mathrm{c}}=0.2 \mathrm{~N} / \mathrm{m}$. As the tip of the small cantilevers is not sharp enough, an electron beam deposited (EBD) tip is grown on the original tip (Fig. 2c) and then sharpened by plasma etching (Fig. 2d).

\subsection{Optical Beam Deflection Detector}

A laser beam is focused onto a small cantilever using a $\times 20$, long-working distance microscope objective lens ${ }^{(3)}$. Its spot size is $2-3 \mu \mathrm{m}$. The laser beam reflected back from the cantilever is collected and collimated by the same objective lens, and guided to a quadrant Si PIN photodiode. The incident and reflected laser beams are separated by a quarter wavelength plate and a polarization beam splitter (Fig. 2e). The outputs from the individual photodiode cells are amplified and conditioned to produce a differential signal proportional to the cantilever deflection and a sum signal proportional to the incident laser power. The bandwidth of the signal conditioner is $20 \mathrm{MHz}$.

\subsection{Fast Amplitude Detector}

The cantilever oscillation amplitude is measured by a Fourier method as follows ${ }^{(4)}$ : The differential signal from the signal conditioner is first digitized with a $100 \mathrm{MHz}$ A/D converter. Then, the Fourier sine and cosine coefficients $(a$ and $b$, respectively) are calculated for the cantilever's first resonant frequency component of the digitized signal, using FPGA and DSP. Finally, $\left(a^{2}+b^{2}\right)^{1 / 2}$ is output as an amplitude signal at every cycle of cantilever oscillation.

\subsection{Fast Scanner}

High-frequency displacement of a piezoactuator produces a large impulse to its supporting mechanism, which is apt to generate unwanted mechanical vibrations. To counteract the impulse and thereby minimize unwanted vibrations, two identical Z-piezoactuators are placed at a supporting base in the opposite direction and displaced simultaneously with the same length ${ }^{(3)}$ (Fig. 2f,g). An alternative method we have recently employed is to hold a piezoactuator at its four rims parallel to the displacement direction so that the center of mass of the piezoactuator is not displaced ${ }^{(7)}$. For the Xscanner, one piezoactuator is sandwiched between two identical flexures so that its center of mass remains stationary
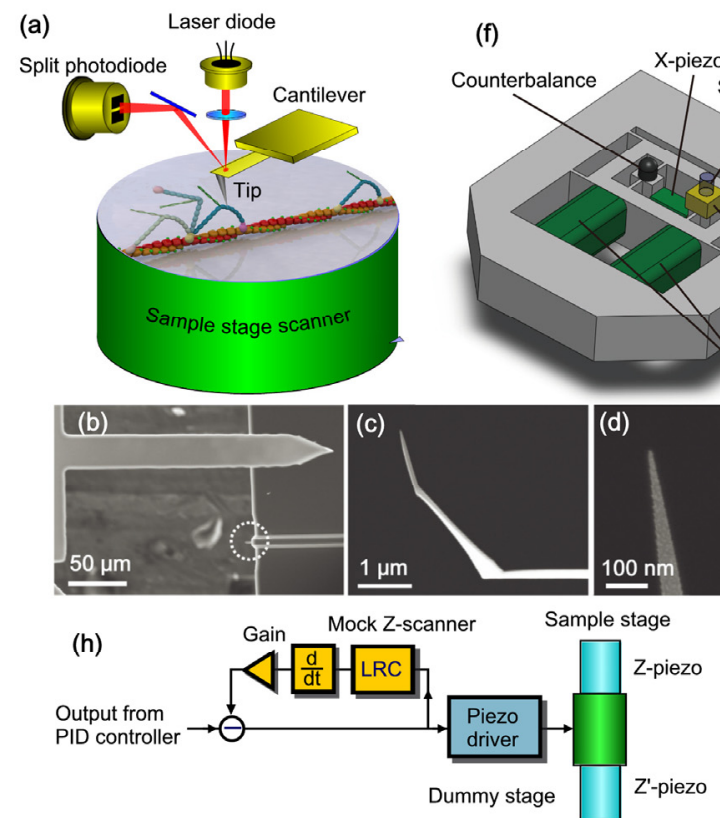
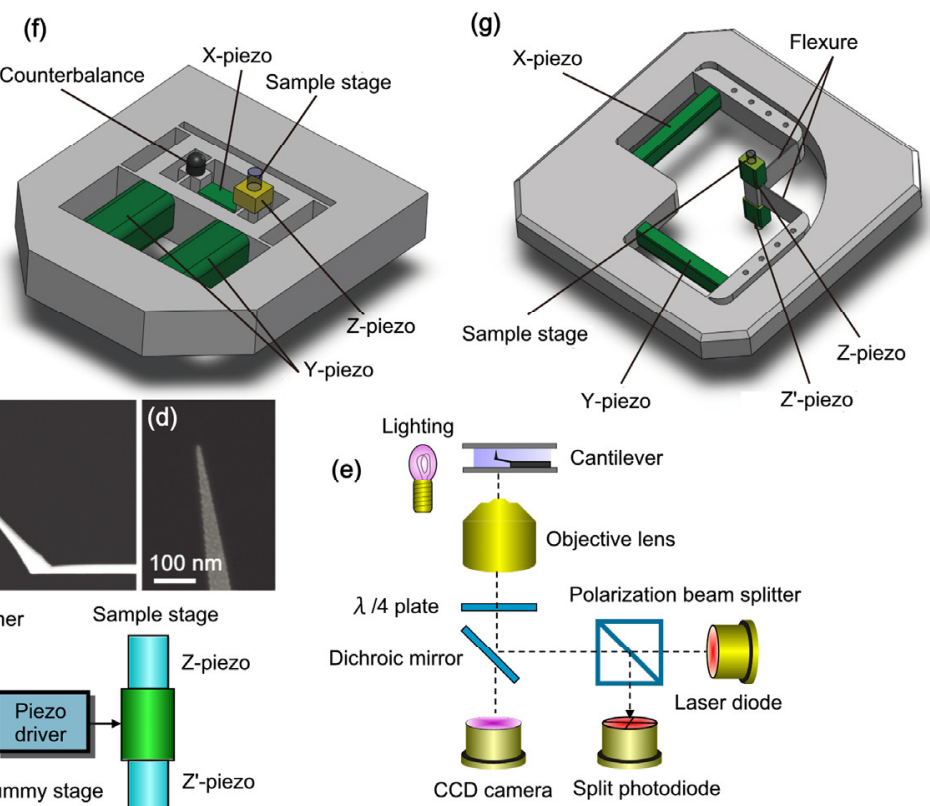

Fig. 2. Various techniques developed for HS-AFM. (a) Schematic of AFM setup. (b) Small cantilever (within the white circle) and conventional one. (c) EBD tip. (d) Sharp tip end of the EBD tip. (e) Optical beam deflection detector for small cantilevers. (f) Fast/narrow-area scanner. (g) Fast/wide-area scanner. (h) Active vibration damping with the use of a mock Z-scanner. 
(Fig. 2f). This design is used for small-area scanners (scan range, $1 \times 4 \times 1 \mu \mathrm{m}^{3}$ for $\mathrm{X}$, Y, and $\mathrm{Z}$ ) to be used to image purified protein molecules. For a wide-area XY-scanner to be used for live cell imaging (scan range, $50 \times 50 \times 3-6 \mu \mathrm{m}^{3}$ for X, Y and Z), the third-class leverage mechanism is employed for displacement magnification ${ }^{(8)}$ (Fig. $2 \mathrm{~g}$ ), together with an inverse feedforward control method for vibration damping ${ }^{(8)}$ (see below).

\subsection{Active Vibration Damping}

In the Z-scanner, unwanted vibrations are not generated from the supporting mechanisms, thanks to the counter balancing method. However, they are generated by the excitation of Z-piezoactuator's first resonant frequency. To supress the vibrations, we can use in principle a feedback Q-control method that mixes the driving signal $F(\mathrm{t})$ with a signal proportional to the velocity of Z-piezoactuator, as indicated by a motion equation: $m \ddot{Z}+\gamma \dot{Z}+k Z=F(t)-\gamma^{\prime} \dot{Z}$. Note that the frictional coefficient increases from $\gamma$ to $\gamma+\gamma$. However, it is difficult to perform high frequency measurements of velocity or displacement of the Z-scanner. Instead of directly measuring the displacement, we use an output signal from a mock Z-scanner ( $L R C$ circuit) that is characterized with the same transfer function as that of the Zscanner $^{(9)}$ (Fig. 2h). This method allows us to eliminate the resonant vibrations completely. Nevertheless, the resonant peak is intentionally remained by $\sim 3 \mathrm{~dB}$ to avoid a significant phase delay. This active damping also works as a means to increase the response speed of the Z-scanner ${ }^{(9)}$. For the X-scanner, we can use an inverse feedforward control method to damp unwanted vibrations as its target displacement is known beforehand ${ }^{(8,10)}$; from the measured frequency response of the X-scanner, $G(i \omega)$, and the Fourier transform of a triangle wave signal to drive the X-scanner, $T(\omega)$, the inverse Fourier transform of $T(\omega) / G(i \omega)$ is calculated beforehand and used as an actual driving signal. For the wide-area $\mathrm{X}$-scanner, we remove high-frequency components from $T(\omega)$ to further suppress unwanted vibrations. With this method, the wide-area X-scanner with the first resonant frequency of $2 \mathrm{kHz}$ can be scanned even at $1 \mathrm{kHz}$ in a nearly triangle trajectory as a function of time ${ }^{(8,10)}$.

\subsection{Dynamic PID Controller}

To solve the parachuting problem, we developed a dynamic PID controller that can change automatically the gain parameters for $\mathrm{P}$ and I components during imaging ${ }^{(11)}$. When a downhill region is being scanned, the cantilever oscillation amplitude $A$ is larger than its set point $A_{\mathrm{s}}$, e.g., $\left(A-A_{\mathrm{s}}\right)>0$. In the dymic PID controller, the gains for P and I components are increased by an amount proportional to $\left(A-A_{\mathrm{s}}\right)$, when $\left(A-A_{\mathrm{s}}\right)$ becomes positive. This method can avoid parachuting even when $A_{\mathrm{s}}$ is set at $0.9 \times A_{0}$, making high-speed imaging compatible with low-invasive imaging.

\section{HS-AFM IMGING OF PROTEIN MOLECULES IN ACTION}

HS-AFM was established in 2008. The feedback bandwidth has now reached $\sim 110 \mathrm{kHz}$. The low-invasive imaging capability has been demonstrated by various imaging studies. For example, a single molecule of $\mathrm{F}_{1}$-ATPase continued to show ATPase reaction-coupled conformational changes during its $40 \mathrm{~s}$ imaging ${ }^{(12)}$. In this period, molecule was tapped more than a few million times with an oscillating tip. The free oscillation amplitude $A_{0}$ of a small cantilever with spring constant $0.2 \mathrm{~N} / \mathrm{m}$ is usually set at $1-2 \mathrm{~nm}$, while the set point amplitude $A_{\mathrm{s}}$ is set at $0.9 \times A_{0}$. Therefore, the average tip force exerted onto the sample, $k_{\mathrm{c}} \times\left(A_{0}-A_{\mathrm{s}}\right) / Q_{\mathrm{c}}$, is in the rage of $10-20 \mathrm{pN}$. Although this magnitude of force sounds large, the mechanical qunatity that affects the sample is not the force itself but the amount of energy transferred from the oscillating cantilever to the sample by tip-sample contact. The cantilever's oscillation energy that is lost at every tip-sample contact, $1 / 2 k_{\mathrm{c}}\left(A_{0}^{2}-A_{\mathrm{S}}^{2}\right) / Q_{\mathrm{c}}$, is $1-4 \times 10^{-20} \mathrm{~J}$ on average $\left(2.4-9.7 k_{\mathrm{B}} T\right.$, where $k_{\mathrm{B}}$ is Boltzmann constant and $T$ is room temperature in Kelvin). This amount of energy is identical to or less than the free energy liberated by hydrolysis of one ATP molecule.

AFM requires the sample be placed on a substrate. AFM can visualize objects only from one direction. Molecules diffusing very fast on the substrate surface cannot be imaged even with HS-AFM. When two molecules to interact with each other are immobilized on a substrate, they have no chance to interact. Therefore, to visualize protein molecules in action, we need to optimize assay conditions, including the substrate surface, buffer solution and sample itself. Under assay conditions appropriately designed for respective proteins, HS-AFM has visualized the molecules in action with great success (see Reviews ${ }^{(13,14)}$ ). Here just one application study on myosin $\mathrm{V}^{(15)}$ is highlighted.

\subsection{Walking Myosin V}

A motor protein, myosin V (M5), moves processively on actin filaments with a step size of $\sim 36 \mathrm{~nm}$, in a hand-overhand manner; that is, the identical two heads, each comprising the motor domain and the neck domain (see Fig. 3a), 
alternately change the leading and trailing roles at every step. These features of M5 motility were deduced mostly from single-molecule optical measurements. However, how the chemical energy of ATP is used during walking was not addressed in the single-molecule studies. This energy usage mechanism has been considered to be basically the same as a widely accepted (but never proven) model that has been put forward for muscle myosins. According to this model, the tension for forward movement of M5 is considered to be generated when Pi is released from the ADP-Pi-bound leading head upon its attachement to actin and this generated tension is consider to drive the forward swing of the leading head when the trailing head is detached from actin upon its binding to ATP. Therefore, the ADP-Pi bound head is supposed to be in a high-energy state, while the ADP-bound head is supposed to be in a low-energy state.

We visualized tail-trancated M5 (M5-HMM) interacting with actin using HS-AFM in order to gain a clue to the chemomechanical coupling in M5. For this visualization, we first prepared mica-supported planar lipid bilayers containing a biotin-lipid. On this surface, partially biotinylated actin filaments were immobilized via streptavidin with a low surface-density. To increase the probability of appearance of actin-bound M5-HMM in a side-on orientation, a positively charged lipid was also included in the lipid bilayers at a low concentration. M5-HMM appeared in AFM images only when it was attached to the immobilized actin filaments, indicating weak non-specific binding of M5-HM onto the substrate surface. Using this assay system, we could observe uni-directionally moving M5-HMM but could not resolve the stepping process because one step occurred very fast (Fig. 3b). To slow down the stepping process, more streptavidin molecules were placed on the substrate as a moderate obstacle for the motion of M5-HMM. Imaging with this method revealed that upon detachment of the trailing head from actin the leading head spontaneously rotated to bring the trailing head forwards and then the trailing head was bound to a frontward actin to become a new leading head (Fig. 3c,d). This observation provided direct evidence that the powerstroke for the forward step of M5-HMM is the rotation of the leading head, which is identical to the swinging lever arm motion hypothesized by Hugh Huxley for muscle myosin.

Besides this anticipated result, HS-AFM movies revealed unexpected behaviors of M5-HMM. In the presence of ATP, the leading head sometimes briefly detached from actin (white triangle, Fig. 3e) and then reattached to actin, while M5-HMM remained at approximately the same position on the filament (third frame in Fig. 3e). We call this behaviour "foot stomp". After foot stomping, the leading head swung forwards following trailing head detachment (the fourth images in Fig. 3e). The leading head that exhibited foot stomping did not carry bound Pi because Pi had already been released since after the head initially attached to actin. This peculiar observation suggests that the ADP-bound
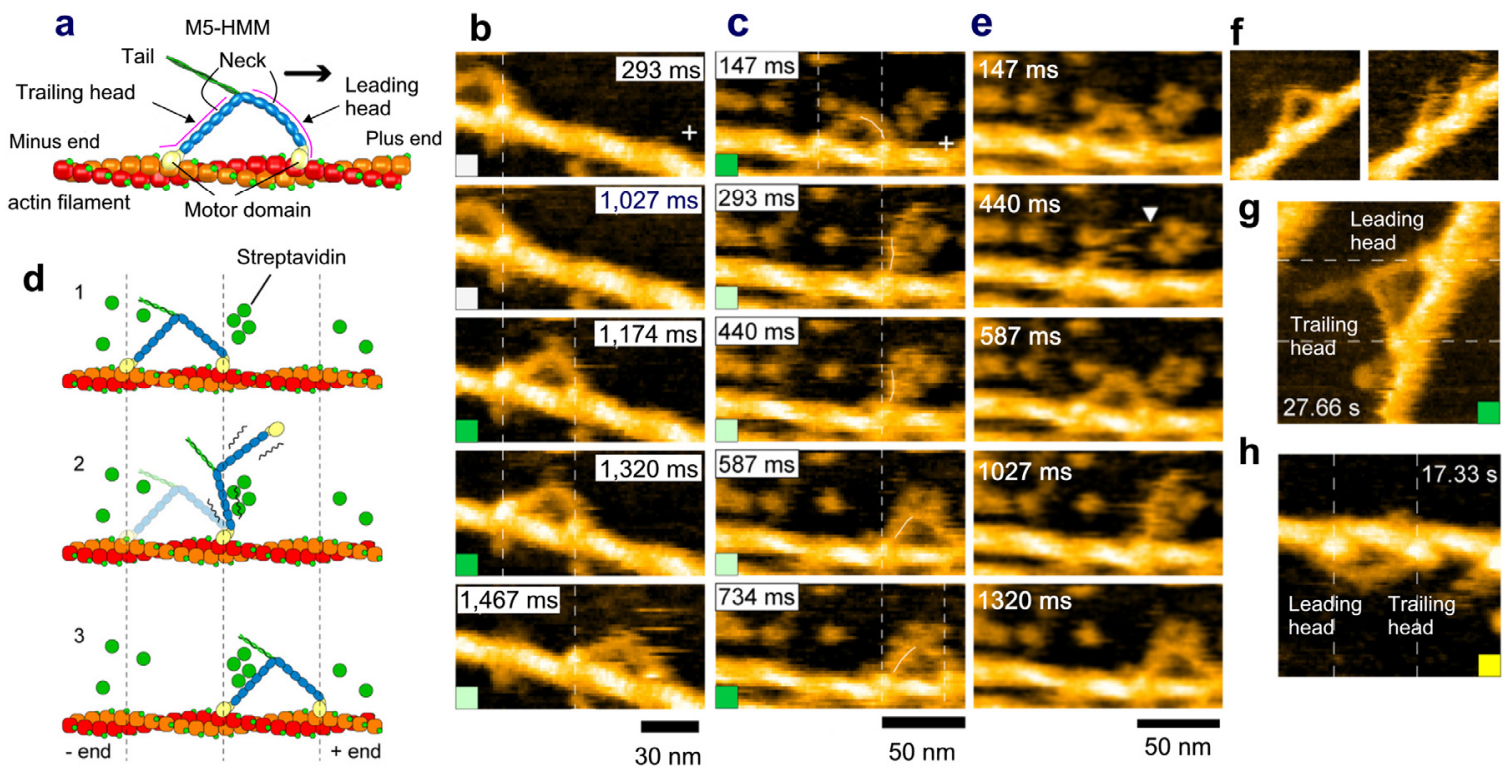

Fig. 3. HS-AFM observation of M5-HMM under various conditions. (a) Schematic of two-headed M5-HMM. (b) HS-AFM images of M5-HMM moving towards the plus end of actin filament. (c) HS-AFM images showing the swinging lever arm motion. (e) HS-AFM images showing foot stomp of the leading head (white triangle in the second image). (f) Unwinding of short coiledcoil tail of two-headed bound M5-HMM in the presence of ADP (left, before unwinding; right, after unwinding). (g) AFM image of two-headed bound M5-HMM in the presence of ADP. (h) AFM image of two headed bound M5-HMM in nucleotide-free condition. 
head can generate tension and execute a powerstroke. This suggestion was reinforced by another observation. In twoheaded bound M5-HMM in the presence of ADP, the short coiled-coil tail was occasionally unwound, upon which the leading head swung forwards (Fig. 3f), very much like a powerstroke observed in the presence of ATP. This observation also suggests that intra-molecular tension can be generated just by binding of the two heads to actin without chemical energy input, and that the lever arm swing of the leading head spontaneously occurs upon trailing head detachment to release this intra-molecular tension. This line of scenario forces us to abandon the well accepted model for the chemomechanical energy transduction in myosin.

The leading head looked nearly straight in the presence of nucleotides (Fig. 3g), whereas it was often kinked in the nucleotide-free condition (Fig. 3h). Therefore, the shape of the leading head can tell us whether or not it is bound to nucleotides. Using this property, we estimated the rate of ADP dissociation from the leading head by observing twoheaded bound M5-HMM in the presence of various concentrations of ADP. The rate constant came out to be $0.1 \mathrm{~s}^{-1}$. That is, ADP dissociates every $10 \mathrm{~s}$ on average. However, M5-HMM makes many steps for $10 \mathrm{~s}$ in the presence of ATP. Therefore, ADP does not dissociate from the leading head when M5-HMM is walking on actin, and hence, only the trailing head can bind ATP to dissociate from actin. This is the mechanism underlying the processive, hand-over-hand movement of M5.

\section{FURTHER PROGRESS OF HS-AFM TECHNOLOGIES}

The HS-AFM system is now being upgraded in various ways (for some of upgrades, see Review ${ }^{(16)}$ ). Tip-scan HS-AFM was recently developed ${ }^{(10)}$, which has a large potential of being combined with various techniques by the utilization of a free space available at the sample side. Tip-scan HS-AFM combined with total internal reflection fluorescence microscopy has already been materialized ${ }^{(10)}$. The interactive mode was recently introduced. This mode allows us to manipulate protein molecules during imaging and look at resulting structure and behavior of the molecules. As seen in these examples, HS-AFM is becoming more useful toolboxes in biological research.

\section{REFERENCES}

[1] Binnig, G,, Quate, C. F. and Gerber, C., “Atomic force microscope,” Phys. Rev. Lett., 56, 930-933 (1986).

[2] Binnig, G., "Force microscopy," Ultramicroscopy, 42-44, 7-15 (1992).

[3] Ando, T., Kodera, N., Takai, E., Maruyama, D., Saito, K. and Toda, A., "A high-speed atomic force microscope for studying biological macromolecules," Proc. Natl. Acad. Sci. USA, 98, 12468-12472 (2001).

[4] Ando, T., Uchihashi, T. and Fukuma, T., "High-speed atomic force microscopy for nano-visualization of dynamic biomolecular processes," Prog. Surf. Sci., 83, 337-437 (2008).

[5] Walters, D. A., Cleveland, J. P., Thomson, N. H., Hansma, P. K., Wendman, M. A., Gurley, G. and Elings, V., "Short cantilevers for atomic force microscopy," Rev. Sci. Instrum., 67, 3583-3590 (1996).

[6] Kitazawa, M., Shiotani, K. and Toda, A., Batch fabrication of sharpened silicon nitride tips, Jpn. J. Appl. Phys. 42, 4844-4847 (2003).

[7] Fukuma, T., Okazaki, Y., Kodera, N., Uchihashi, T. and Ando, T., "High resonance frequency force microscopy scanner using inertia balance support," Appl. Phys. Lett., 92, 243119 (2008).

[8] Watanabe, H., Uchihashi, T., Kobashi, T., Shibata, M., Nishiyama, J., Yasuda, R. and Ando, T., "Wide-area scanner for highspeed atomic force microscopy," Rev. Sci. Instrum., 84, 053702 (2013).

[9] Kodera, N., Yamashita, H. and Ando, T., "Active damping of the scanner for high-speed atomic force microscopy," Rev. Sci. Instrum., 76, 053708 (2005).

[10] Fukuda, S., Uchihashi, T., Iino, R., Okazaki, Y., Yoshida, M., Igarashi, K. and Ando, T., "High-speed atomic force microscope combined with single-molecule fluorescence microscope," Rev. Sci. Instrum., 84, 073706 (2013).

[11] Kodera, N., Sakashita, M. and Ando, T., "Dynamic proportional-integral-differential controller for high-speed atomic force microscopy," Rev. Sci. Instrum., 77, 083704 (2006).

[12] Uchihashi, T., Iino, R., Ando, T. and Noji, H., "High-speed atomic force microscopy reveals rotary catalysis of rotorless $F_{1}-$ ATPase," Science, 333, 755-758 (2011).

[13] Ando, T., Uchihashi, T. and Kodera, N., "High-speed AFM and applications to biomolecular systems," Annu. Rev. Biophys., 42, 393-414(2013).

[14] Ando, T., Uchihashi, T. and Scheuring, S., "Filming biomoleculear processes by high-speed atomic force microscopy", Chem. Rev., 114, 3120-3188 (2014).

[15] Kodera, N., Yamamoto, D., Ishikawa, R. and Ando, T., "Video imaging of walking myosin V by high-speed atomic force microscopy," Nature, 468, 72-76 (2010).

[16] Uchihashi, T., Watanabe, H., Fukuda, S., Shibata, M. and Ando, T., "Functional extension of high-speed atomic force microscopy," Ultramicroscopy, 160, 182-196 (2016). 